\title{
\begin{tabular}{l|l} 
Mitraries & DSpace@MIT
\end{tabular}
}

\author{
MIT Open Access Articles
}

\section{Patterning nano-domains with orthogonal functionalities: Solventless synthesis of self-sorting surfaces}

The MIT Faculty has made this article openly available. Please share how this access benefits you. Your story matters.

Citation: Sung Gap Im et al. "Patterning nano-domains with orthogonal functionalities:

Solventless synthesis of self-sorting surfaces." Optical Data Storage Topical Meeting, 2009. ODS '09. 2009. 73-75. (c)2009 Institute of Electrical and Electronics Engineers.

As Published: http://dx.doi.org/10.1109/0DS.2009.5031758

Publisher: Institute of Electrical and Electronics Engineers

Persistent URL: http://hdl.handle.net/1721.1/59421

Version: Final published version: final published article, as it appeared in a journal, conference proceedings, or other formally published context

Terms of Use: Article is made available in accordance with the publisher's policy and may be subject to US copyright law. Please refer to the publisher's site for terms of use. 


\title{
Patterning Nano-Domains with Orthogonal Functionalities: Solventless Synthesis of Self-Sorting Surfaces
}

\author{
Sung Gap Im, Byeong-Su Kim, Ki Wan Bong, Salmaan H. Baxamusu, Wyatt E. Tenhaeff, \\ Patrick S. Doyle, Paula T. Hammond, and Karen K. Gleason (kkg@mit.edu) \\ Department of Chemical Engineering and Institute for Soldier Nanotechnologies, \\ Massachusetts Institute of Technology, Cambridge, Massachusetts 02139
}

\begin{abstract}
Vapor deposited functional polymer thin films can undergo rapid covalent functionalization. Patterning of two functional layers displaying orthogonal reactivity enables sorting of aqueous mixtures of dyes and nanoparticles, such as quantum dots, onto selective areas of nanopatterned surfaces.
\end{abstract}

\section{Introduction}

Surfaces covered by well-defined organic functional groups can serve as platforms to immobilize molecular species such as fluorescent dyes or peptides, as well as particles, including quantum dots and metal nanoparticles. Reaction with the organic functional group is desired to provide high selectivity and strong binding of the desired component onto the synthetic surface. In contrast, physisorption to the surface is undesirable because it is generally nonspecific to the binding site.

Immobilization schemes for molecules and particles combined with micropatterning techniques promise to be enabling methods for novel device fabrication. However, the solvents employed in conventional patterning techniques often result in dissolution or delamination of the functional layer. Additionally, the solvents and/or irradiation methods employed by traditional patterning schemes often degrade the activity of the desired organic functional groups.

The current work reports the vacuum deposition of polymeric surfaces having highly reactive acetylene functional groups. These $-\mathrm{C} \equiv \mathrm{CH}$ are desired because of their ability to quickly and selectively create interfacial covalent linkages via Huisgen 1,3-dipolar cycloaddition. Using this so-called "click chemistry", azide species rapidly form robust attachments to the surface in a wide range of solvents, including water. These reactions are catalyzed by $\mathrm{Cu}(\mathrm{I})$, occurs at mild temperatures $\left(25-70{ }^{\circ} \mathrm{C}\right)$ and with few side reactions.

Additionally, micro- and nano- patterning processes are demonstrated which retain the reactivity of the $-\mathrm{C} \equiv \mathrm{CH}$ surface groups. Finally, a facile solventless method for synthesizing nanopatterned bifunctional surfaces is also reported.

\section{Results and Discussion}

A click chemistry active polymer coating was prepared by the novel one-step synthetic process of initiated chemical vapor deposition (iCVD) using the commercially available monomer propargyl methacrylate (PMA). A surface treatment was developed to chemically graft the iCVD pPMA onto various inorganic substrates, providing the chemical and mechanical stability required for surviving the subsequent wet chemical steps required for subsequent click reactions. Spectroscopic identification of $\mathrm{C} \equiv \mathrm{C}$ bonding and successful demonstration of click chemistry confirms that the highly reactive pendant acetylene functionalities survived the iCVD film deposition process. The iCVD pPMAbased films exhibits electron beam sensitivity and hence can be directly patterned via e-beam 
lithography without requiring use of a conventional resist layer. A well-defined $200 \mathrm{~nm}$ line and space pattern was obtained with a dosage of $50 \mathrm{mC} \cdot \mathrm{cm}^{2}$ at $50 \mathrm{keV}$ acceleration voltage. These patterned surfaces were selectively conjugated with fluorescent dyes and quantum dots (Fig. 1).

A dual-functional click-active/amine patterned surface was obtained using an iCVD of the polymer coating of poly (propargyl methacrylate) (PPMA) grown onto of a poly allylamine (PAAm) film deposited by via plasma polymerization process. Capillary force lithography (CFL) process was applied to the stacked PPMA on PAAm layer structure. Crosslinking allows the bottom layer to remain immobile during the CFL step. Thus, the CFL selectively patterned the top PPMA layer while maintaining bottom PAAm blanket layer intact, thus yielding multifunctional nanopatterns (Fig. 2).

The minimum feature size of this bifunctional nano-pattern was $110 \mathrm{~nm}$. The entire fabrication process is solventless and low temperature, enabling the retention of reactive surface functionalities. In contrast to conventional photolithography, CFL does not require resists, developers, solvents, or chemically destructive irradiation can deactivate the reactive functional groups.

The nanodomains containing surface amine groups which can be functionalized by carbodiimide chemistry with $N$-hydroxysuccinimide (NHS). The click and NHS-reactions are highly orthogonal to each other so that non-specific immobilization can be minimized. These advantageous characteristics enable the covalent functionalization of two independent components in a one-pot functionalization process in self-recognized fashion. The one-pot orthogonal functionalization was sucessfully performed in an aqueous solution at room temperature (Fig. 3).

\section{Conclusions}

Click chemistry active surfaces were successfully deposited by iCVD. Bilayers of click-active iCVD films on top of PECVD amine-containing films were patterned by capillary force lithography to obtain dual functional nano-patterned surfaces having a minimum feature size of $110 \mathrm{~nm}$. Exposing the patterned surface to a mixture of two fluorescent dyes resulted in self-sorting of the dyes via conjugation of the dyes onto selected areas of the pattern. The characteristics of the iCVD thin film growth method include the retention of reactive organic functional groups, compatibility with conventional silicon device and MEMS fabrication schemes, the ability to produce thin films which are conformal over micro- and nano-features, and facile nanopatterning schemes. We anticipate these features will make iCVD a powerful integration platform for organic and nanoparticle functionalization into next generation devices.

\section{Acknowledgment}

This research was supported by the U.S. Army through the Institute for Soldier Nanotechnologies, under Contract DAAD-19-02-D-0002 with the U.S. Army Research Office. 

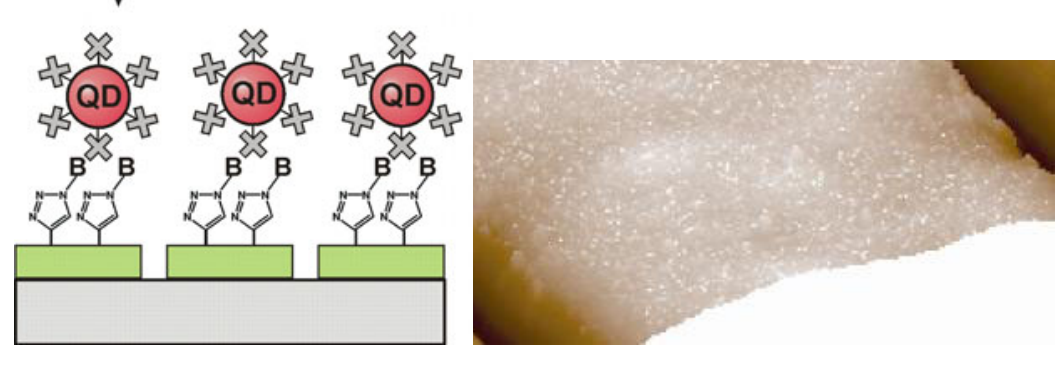

Fig. 1. (left) Schematic of quantum dot (QD) immobilization onto the e-beam patterned iCVD polymer film achieved through biotinstreptavidin binding by click chemistry. (right) Atomic Force Micrograph (AFM) of QDs conjugated onto 2 micron wide line of iCVD polymer film.
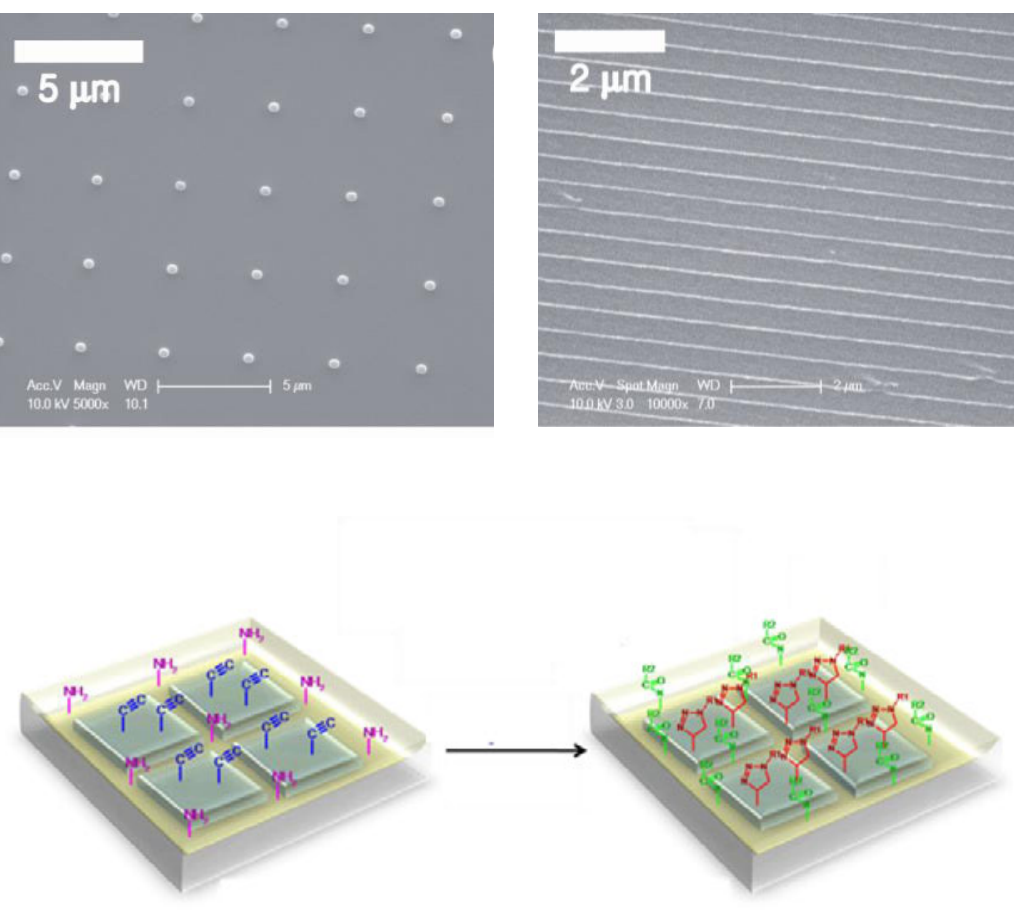

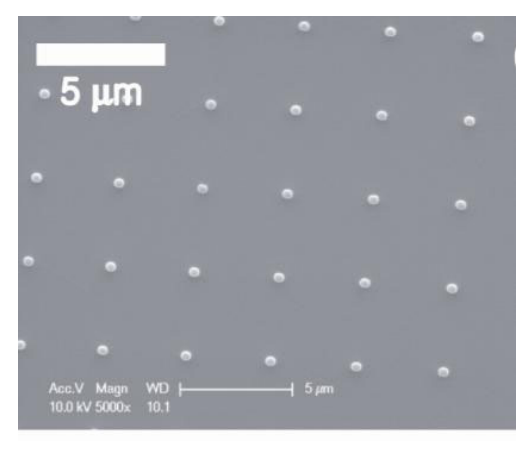

Fig. 2. Scanning electron micrographs (SEM) of (left) a $250 \mathrm{~nm}$ dot pattern and (right) a $110 \mathrm{~nm}$ line and space pattern of iCVD PPMA achieved by capillary force lithography. The underlying material is a blanket film of plasma deposited PAAm, which remains immobile because of crosslinking. The $-\mathrm{C} \equiv \mathrm{CH}$ groups of the PPMA can undergo click chemistry while the amine groups of the PAAm can be functionalized by the NHS reactions.

\section{Fig. 3.}

(top) Schematic of a bifunctional surface showing (left) patterned square areas of acetylenic groups $(-\mathrm{C} \equiv \mathrm{CH})$ on top of a blanket films with surface amine groups $\left(-\mathrm{NH}_{2}\right)$; (right) Area selective binding of red and green dyes. The red dye is attached by the acetylenic groups through click chemistry while the green dye is attached by the surface amine groups using NHS functionalization.
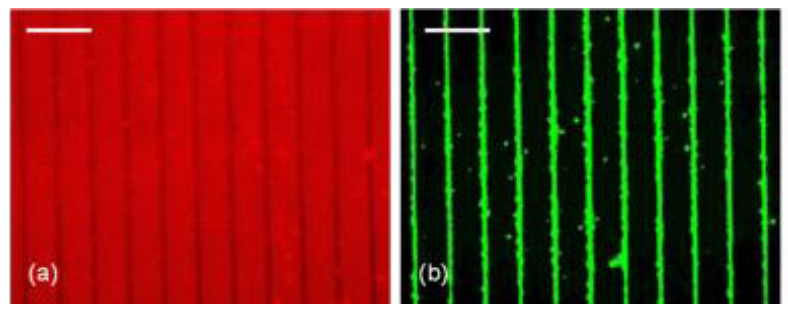

(bottom) Fluorescence micrographs after one-pot functionalization of bifunctional line and space pattern from a mixture of red and green dyes of showing selective attachment of the (a) red dye which is excited at $545 \mathrm{~nm}$ and (b) the green dye which excited at $491 \mathrm{~nm}$; (c) Overlapped image of (a) and (b). Each scale bars represents 30 microns 